\title{
Impact of Total Occlusion of an Infarct-Related Artery on Long-Term Mortality in Acute Non-ST-Elevation Myocardial Infarction Patients Who Underwent Early Percutaneous Coronary Intervention
}

\author{
Min Chul Kim, ${ }^{1}$ MD, Youngkeun Ahn, ${ }^{1}$ MD, Shi Hyun Rhew, ${ }^{1}$ MD, Myung Ho Jeong,,${ }^{1}$ MD, \\ Ju Han KIm, ${ }^{1}$ MD, Young Joon Hong, ${ }^{1}$ MD, Shung Chull ChaE, ${ }^{2}$ MD, Young Jo KIM, ${ }^{3}$ MD, \\ Seung Ho Hur, ${ }^{4}$ MD, In Whan SeOng, ${ }^{5}$ MD, Jei Keon ChaE, ${ }^{6}$ MD, for the KAMIR Investigators
}

\section{SUMMARY}

Some patients with non-ST-elevation myocardial infarction (NSTEMI) have a total occlusive infarct-related artery. However, the long-term prognosis of these patients is uncertain, particularly for those who underwent an early invasive strategy.

The aim of this study was to determine the clinical impact of total occlusion (TO) of an infarct-related artery (IRA) in these patients.

A total of 2,094 patients with NSTEMI who underwent an early invasive strategy with percutaneous coronary intervention (PCI) in the Korea Acute MI Registry (KAMIR) were analyzed (TO group; 665 patients, and non-TO group; 1,429 patients).

In-hospital and one-year clinical outcomes were compared between the two groups. The left circumflex $(42.9 \%)$ and right coronary artery (31.9\%) were the major IRA in the TO group, while the left anterior descending artery was more common as an IRA in the non-TO group (44.1\%). In-hospital complications including death and cardiogenic shock occurred frequently in the TO group. Also, the rates of one-month and 12-month adverse cardiac outcomes were higher in the TO group. In the Cox-proportional hazard model, TO in IRA predicted 12-month all-cause death.

In conclusion, NSTEMI patients with TO in IRA showed worse short- and long-term clinical outcomes compared with those of non-TO patients. (Int Heart J 2012; 53: 160-164)

Key words: Non-ST-elevation myocardial infarction, Total occlusion, Percutaneous coronary intervention

A mong patients with acute myocardial infarction (AMI), non-ST-elevation MI (NSTEMI) is a heterogenous group in aspects of diagnosis, treatment, and timing of percutaneous coronary intervention (PCI). It is difficult to decide the optimal timing of PCI in patients with NSTEMI because of various clinical settings. However, an early invasive strategy with PCI gives better clinical and angiographic results in high risk patients with NSTEMI than conservative management or late PCI. ${ }^{1,2}$

Generally, more than $50 \%$ of patients diagnosed as STEMI are known to have total occlusion (TO) of an infarct-related artery (IRA). ${ }^{2)}$ However, it is difficult to predict TO in patients with NSTEMI. One study regarding this issue revealed that the probability of TO in patients with NSTEMI was proportional to the time from symptoms to the diagnostic coronary angiogram. ${ }^{3)}$ This finding is perhaps associated with a progression of nonocclusion to occlusion in IRA with time. In 2 recent studies, the proportion of TO of culprit artery in patients with
NSTEMI was about one quarter, and TO was associated with larger infarcts and worse clinical outcomes. ${ }^{4,5)}$

Therefore, we assessed the clinical characteristics, angiographic findings, and long-term clinical outcomes in patients with NSTEMI having TO of the culprit artery in the largescaled Korea AMI Registry (KAMIR), which should provide valuable information about this issue.

\section{Methods}

Study design and populations: A total of 2,094 patients in the KAMIR from November 2005 to January 2008 were included in the present study. The KAMIR is a prospective, observational, multicenter registry reflecting current practices of management, risk factors, and clinical outcomes in Korean patients with AMI. The KAMIR includes patients with both STEMI and NSTEMI. The 50 hospitals with facilities for primary PCI

From ${ }^{1}$ Chonnam National University Hospital, Gwangju, ${ }^{2}$ Kyungpook National University Hospital, ${ }^{3}$ Yeungnam University Hospital, ${ }^{4}$ Keimyung University Hospital, Daegu, ${ }^{5}$ Chungnam National University Hospital, Daejon, and ${ }^{6}$ Chonbuk National University Hospital, Jeonju, Korea.

This work was supported by a grant from the National Research Foundation of Korea funded by the Korean Government (MEST), Republic of Korea (2010-0020261), and by a grant from the Korea Healthcare Technology R\&D Project, Ministry for Health, Welfare \& Family Affairs, Republic of Korea (A084869).

Address for correspondence: Youngkeun Ahn, MD, Department of Cardiology, Cardiovascular Center, Chonnam National University Hospital, 671 Jaebongro, Donggu, Gwangju 501-757, Korea.

Received for publication December 22, 2011

Revised and accepted April 13, 2012. 
all participated, and each ethics committee approved the study protocol. Among 13,897 patients in the registry, NSTEMI patients who underwent early invasive strategy with PCI were analyzed. All patients underwent PCI within 3 days after admission. Coronary flow before and after PCI was classified by the Thrombolysis In Myocardial Infarction (TIMI) score. ${ }^{6}$ Patients with no data concerning symptom-to-PCI time and preprocedural TIMI flow grade were excluded from the analysis. The patients were divided into two groups based on the presence of pre-TIMI flow grade zero; pre-TIMI flow 0 (TO group, $62.4 \pm 12.2$ years, $n=665$ ) and pre-TIMI flow $\geq 1$ (non-TO group, $62.8 \pm 12.1$ years, $n=1,429)$. Among these patients, $1,934(92.4 \%)$ were followed-up at one-month and 1,725 (82.4\%) completed 12-month clinical follow-up.

Measurements: Typical chest pain was defined as any combination of these features; sensations in the chest of squeezing, heaviness, pressure, weight, vise-like aching, burning, tightness, radiation to shoulder, neck, jaw, inner arm, epigastrium, and symptoms abate when stressor is gone or nitroglycerin is taken. The diagnosis of AMI was based on clinical presentations, increased cardiac biomarkers (creatine kinase-MB, troponin-I or troponin-T), and 12-lead electrocardiographic findings. ${ }^{7)}$ The TIMI risk score to predict short term risks in patients with NSTEMI and the rate of patients with high risk (TIMI risk score $\geq 5$ ) were calculated. ${ }^{8)}$ All laboratory findings were obtained immediately after admission. Coronary arterial lesion type was determined according to the American College of Cardiology/American Heart Association (ACC/AHA) classification. ${ }^{9)}$ The culprit vessel of patients with NSTEMI was identified based on the findings of coronary angiography, 12lead electrocardiogram, 2-dimensional echocardiogram, and noninvasive stress test, if possible. Multivessel disease was defined as $\geq 70 \%$ diameter stenosis in at least 2 major epicardial coronary arteries. The diameter, length, and number of implanted stents were those of the culprit artery. Left ventricular ejection fraction was checked by 2-dimension echocardiography before discharge. All patients received $300 \mathrm{mg}$ aspirin and 300 to $600 \mathrm{mg}$ clopidogrel as a loading dose before PCI. A 50 to $70 \mathrm{U} / \mathrm{kg}$ dose of unfractionated heparin was used before or during PCI to maintain an activated clotting time of 250 to 300 seconds. After PCI, 100 to $300 \mathrm{mg}$ aspirin and $75 \mathrm{mg}$ clopidogrel were prescribed daily as a maintenance dose for AMI. Glycoprotein IIb/IIIa inhibitor use and insertion of an intraaortic balloon pump (IABP) in patients with cardiogenic shock or hypotension were left to the discretion of the operator.

Study outcomes: Baseline clinical characteristics, laboratory findings, and management methods were analyzed. In-hospital outcomes including periprocedural complications (including cardiogenic shock, ventricular tachycardia or fibrillation, major bleeding, and atrioventricuar block needing temporary pacemaker) were evaluated. Clinical follow-up was performed for 12 months. Endpoints included major adverse cardiac events [MACEs, all-causes of death, recurrent MI, and repeated revascularization (PCI or surgical revascularization)]. Repeated PCI consisted of target lesion revascularization (TLR), target vessel revascularization (TVR), and non-TVR. Staged-PCI during initial admission was not included in repeated PCI. Recurrent MI was defined as recurrent symptoms with new electrocardiographic changes reflecting MI or increased cardiac biomarkers at least twice the upper normal limit. ${ }^{10)}$ TLR was defined as PCI for restenosis or other lesion complication that was treated in a segment $5 \mathrm{~mm}$ proximal or distal to a stent. TVR was defined as repeated PCI for any coronary artery segment proximal or distal to the target lesion. ${ }^{11)}$

Statistical analyses: The statistical analyses were performed using SPSS for Windows, version 17.0 (Statistical Package for the Social Sciences, SPSS Inc., USA). Continuous variables are presented as the mean \pm standard deviation and were compared using Student's $t$-test between two groups. Categorical variables were analyzed using the chi-square test to determine the significance of differences. All variables considered relevant in TO of IRA in patients with NSTEMI were included to identify associated factors using logistic regression. They were age, symptom-to-PCI time, TIMI risk score $\geq 5$, gender, typical chest pain at admission, Killip class $\geq 3$, electrocardiographic abnormalities (ST-deviation $\geq 0.5 \mathrm{~mm}$ and T-inversion) on inferolateral leads (leads $\mathrm{V}_{5}, \mathrm{~V}_{6}$, I, aVL, II, III, and aVF), prior coronary artery disease (CAD), hypertension, diabetes mellitus, dyslipidemia, current or former smoker, familial history of CAD, left ventricular ejection fraction $\leq 40 \%$, serum creatinine, and maximal troponin-I level. Cox proportional hazard model analysis adjusted with co-variables showed significance in univariate analysis ( $P$ value less than 0.1$)$ for mortality and the other variables that have been reported to be associated with the prognosis of patients with AMI was used to compare 12-month mortality between two groups. All analyses were 2-tailed and all variables were considered significant when $P$ was less than 0.05 .

\section{Results}

Baseline characteristics: Baseline clinical characteristics and laboratory findings are shown in Table I. Mean age was similar

Table I. Baseline Clinical Characteristics and Laboratory Findings

\begin{tabular}{|c|c|c|c|}
\hline & $\begin{array}{c}\text { TO } \\
(n=665)\end{array}$ & $\begin{array}{c}\text { Non-TO } \\
(n=1,429)\end{array}$ & $P$ \\
\hline Age (years) & $62.39 \pm 12.2$ & $62.84 \pm 12.1$ & 0.437 \\
\hline Male gender, $n(\%)$ & $462(69.5)$ & 1005 (70.4) & 0.682 \\
\hline $\begin{array}{l}\text { Typical chest pain at admission, } \\
n(\%)\end{array}$ & $564(84.8)$ & $1202(84.4)$ & 0.846 \\
\hline TIMI risk score & $2.49 \pm 1.23$ & $2.48 \pm 1.27$ & 0.879 \\
\hline TIMI risk score $\geq 5, n(\%)$ & $31(4.7)$ & $92(6.4)$ & 0.111 \\
\hline $\begin{array}{l}\text { Killip class III/IV on admission, } \\
\quad n(\%)\end{array}$ & $37(5.6)$ & $91(6.5)$ & 0494 \\
\hline Prior history of CAD, $n(\%)$ & $135(20.4)$ & 267 (18.7) & 0.404 \\
\hline Hypertension, $n(\%)$ & $321(48.5)$ & $757(53.0)$ & 0.054 \\
\hline Diabetes mellitus, $n(\%)$ & $167(25.2)$ & 427 (29.9) & 0.029 \\
\hline Smoking, $n(\%)$ & $395(59.5)$ & $805(56.5)$ & 0.217 \\
\hline Dyslipidemia, $n(\%)$ & $100(15.1)$ & $172(12.1)$ & 0.059 \\
\hline Familial history of CAD, $n(\%)$ & $64(9.7)$ & $116(8.2)$ & 0.276 \\
\hline Serum creatinine (mg/dL) & $1.18 \pm 1.3$ & $1.13 \pm 1.3$ & 0.441 \\
\hline Troponin-I (mg/dL) & $25.7 \pm 52.5$ & $26.9 \pm 57.2$ & 0.676 \\
\hline CK-MB (mg/dL) & $107.7 \pm 381.7$ & $93.3 \pm 278.1$ & 0.388 \\
\hline N-terminal pro BNP (pg/mL) & $2379.8 \pm 6344.4$ & $1711.7 \pm 4483.3$ & 0.034 \\
\hline High sensitivity CRP (mg/dL) & $14.1 \pm 60.4$ & $14.4 \pm 62.0$ & 0.935 \\
\hline Baseline left ventricular EF (\%) & $55.5 \pm 11.4$ & $55.2 \pm 11.6$ & 0.567 \\
\hline
\end{tabular}

TO indicates total occlusion; Non-TO, non-total occlusion; TIMI, thrombolysis in myocardial infarction; CAD, coronary artery disease; CK-MB, creatine kinase-myocardial band isoenzyme; BNP, B-type natriuretic peptide; CRP, C-reactive protein; and EF, ejection fraction. 
and there was no gender difference between the 2 groups. Also, the admission Killip class and TIMI risk score were not different and the incidences of typical chest pain were similar. Among known cardiovascular risk factors, only the incidence of diabetes was higher in the non-TO group. For other risk factors such as cerebrovascular disease, prior heart failure, and peripheral vascular disease, there was no significant difference (data not shown). Left ventricular ejection fraction by 2-dimension echocardiography did not reveal any difference in the 2 groups. There were no significant differences in laboratory findings, except for the level of N-terminal pro B-type natriu-

Table II. Coronary Angiographic Findings and Procedural Results

\begin{tabular}{lccr}
\hline & TO & Non-TO & \multicolumn{1}{c}{$P$} \\
& $(n=665)$ & $(n=1,429)$ & \multicolumn{1}{c}{} \\
\hline Symptom-to-PCI time (days) & $1.26 \pm 0.84$ & $1.22 \pm 0.79$ & 0.276 \\
ACC/AHA lesion type B2/C, $n(\%)$ & $532(83.4)$ & $983(71.8)$ & $<0.001$ \\
Multivessel disease, $n(\%)$ & $398(59.9)$ & $900(63.1)$ & 0.175 \\
PCI with coronary stenting, $n(\%)$ & $572(86.0)$ & $1335(93.4)$ & $<0.001$ \\
$\quad$ Drug-eluting stent, $n(\%)^{*}$ & $521(91.6)$ & $1217(91.9)$ & 0.785 \\
$\quad$ Stent length (mm) & $25.1 \pm 6.4$ & $24.3 \pm 6.5$ & 0.012 \\
$\quad$ Stent diameter (mm) & $3.04 \pm 0.4$ & $3.14 \pm 0.43$ & $<0.001$ \\
$\quad$ Total number of implanted stents & $1.58 \pm 0.89$ & $1.65 \pm 0.93$ & 0.125 \\
PCI success rate, $n(\%)$ & $611(94.3)$ & $1370(98.3)$ & $<0.001$ \\
Cardiopulmonary resuscitation, $n$ & & & \\
$\quad(\%)$ & $12(1.8)$ & $17(1.2)$ & 0.315 \\
Mechanical ventilation, $n(\%)$ & $14(2.1)$ & $20(1.4)$ & 0.265 \\
IABP insertion, $n(\%)$ & $19(2.9)$ & $25(1.8)$ & 0.104 \\
\hline
\end{tabular}

TO indicates total occlusion; Non-TO, non-total occlusion; PCI, percutaneous coronary intervention; and IABP, intraaortic ballooning pump. "Drug-eluting stent implantation rate was calculated in patients who underwent stenting.

A

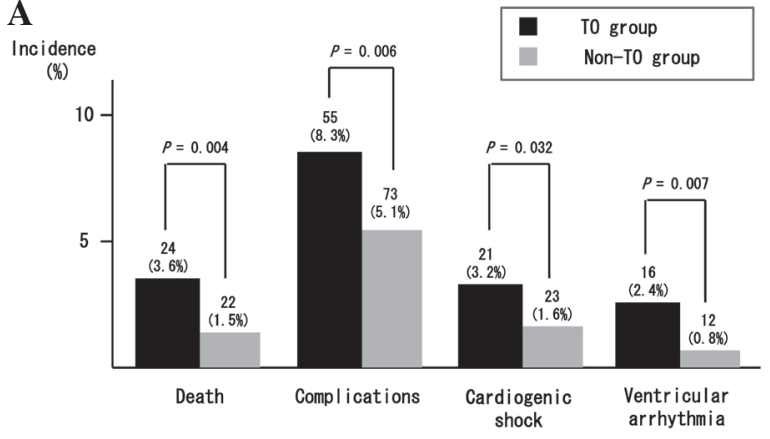

B

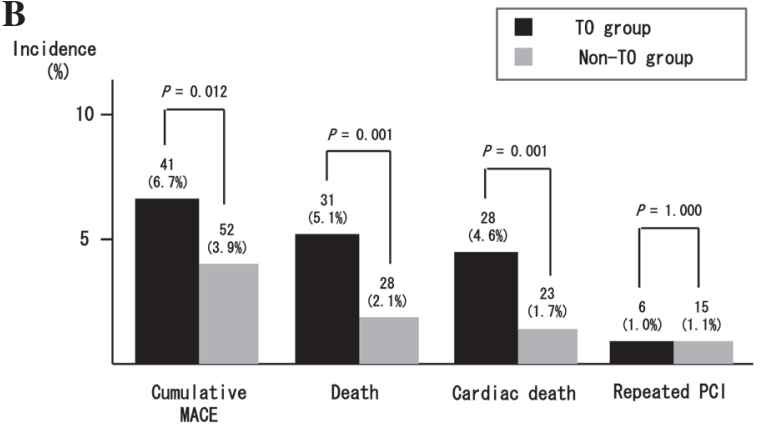

retic peptide. The prescription rate of evidence-based medications associated with improvement of prognosis (defined as antiplatelet drugs, beta-blockers, angiotensin converting enzyme inhibitors or angiotensin-II receptor antagonists, and statins) was similar between both groups during hospitalization and at discharge. ${ }^{12)}$ Also, the frequencies of use of glycoprotein IIb/IIIa inhibitors, antithrombotic drugs, and diuretics were similar (data not shown).

Procedural findings: The distribution of IRA in the 2 groups was as follows; TO group: left-anterior descending $24.5 \%$, right coronary artery $31.9 \%$, and left circumflex artery $42.9 \%$,

Table III. Independent Predictors of TO in Infarct-Related Artery

\begin{tabular}{llr}
\hline & $\begin{array}{c}\text { Odd ratio } \\
\text { (95\% confidence } \\
\text { interval) }\end{array}$ & $P$ \\
\hline Symptom-to-PCI time & $1.04(0.90$ to 1.19$)$ & 0.598 \\
TIMI risk score $\geq 5$ & $0.69(0.42$ to 1.16$)$ & 0.162 \\
Male gender & $0.98(0.76$ to 1.26$)$ & 0.847 \\
Typical symptom at admission & $1.18(0.86$ to 1.62$)$ & 0.318 \\
Killip class $\geq 3$ & $1.08(0.68$ to 1.71$)$ & 0.755 \\
EKG abnormalities on inferolateral leads & $1.53(1.21$ to 1.93$)$ & $<0.001$ \\
Prior coronary artery disease & $1.31(0.98$ to 1.75$)$ & 0.065 \\
Hypertension & $0.85(0.67$ to 1.08$)$ & 0.192 \\
Dyslipidemia & $1.45(1.04$ to 2.02$)$ & 0.028 \\
Serum creatinine & $1.10(0.99$ to 1.22$)$ & 0.067 \\
Maximal troponin-I & $0.99(0.99$ to 1.01$)$ & 0.111 \\
LV ejection fraction $\leq 40 \%$ & $0.83(0.57$ to 1.21$)$ & 0.330 \\
\hline
\end{tabular}

TO indicates total occlusion; PCI, percutaneous coronary intervention; TIMI, Thrombolysis in Myocardial Infarction; EKG, electrocardiogram; and $\mathrm{LV}$, left ventricle.

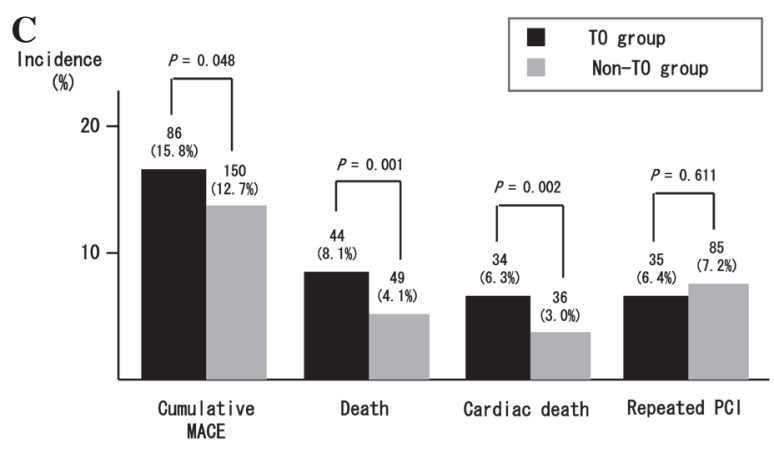

Figure 1. Cumulative clinical outcomes. A: In-hospital events, B: onemonth clinical outcomes, and C: 12-month clinical outcomes. 


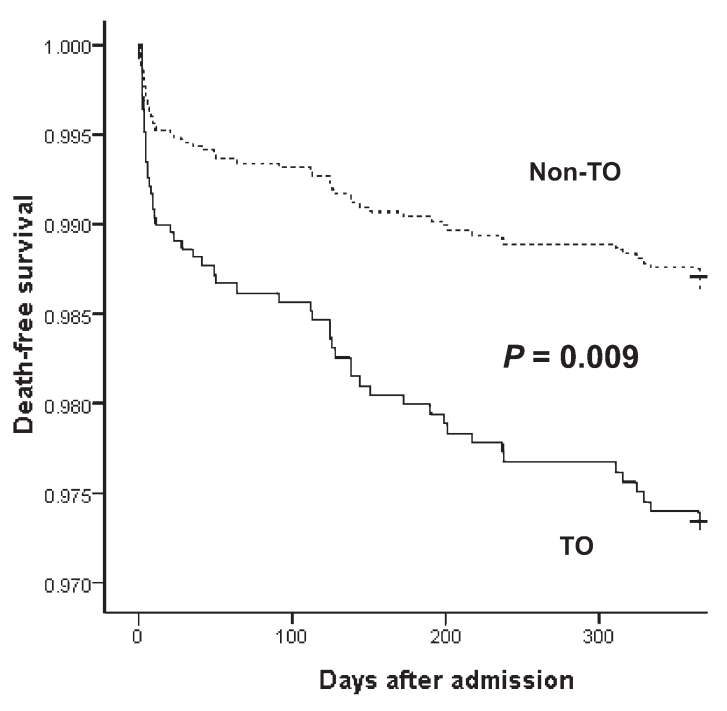

Figure 2. 12-month death-free survival rate in patients with NSTEMI with TO of culprit artery.

non-TO group: left-anterior descending $44.1 \%$, right coronary artery $24.7 \%$, and left circumflex artery $28.5 \%$. In the coronary angiographic findings and procedural results, the symptom-toPCI time was similar and the frequency of multivessel disease was not different between the groups. More complex lesions $\left(\mathrm{B}_{2} / \mathrm{C}\right.$ lesion in ACC/AHA definition) were observed in the TO group. Although coronary stenting was performed more frequently in the non-TO group, the frequency of drug-eluting stent implantation was similar between the 2 groups. The PCI success rate was higher in the non-TO group. The frequencies of cardiopulmonary resuscitation, implantation of IABP, and mechanical ventilation performed during the procedure were similar (Table II).

Multivariate analysis with logistic regression showed 12lead electrocardiographic abnormalities in the inferolateral leads and a history of dyslipidemia were associated with TO in an IRA on diagnostic coronary angiograms (Table III).

Clinical outcomes: In-hospital, one-month, and 12-month clinical outcomes are shown in Figure 1. The incidence of periprocedural complication development including cardiogenic shock and ventricular tachycardia or fibrillation was higher in the TO group. Also, in-hospital mortality was higher in the TO group. Composite endpoints occurred in 93 patients $(4.8 \%)$ at one-month and 236 patients $(13.7 \%)$ at 12-months. At one-month, MACE and all-cause death were greater in the TO-group, and at 12-months, the MACE rate and mortality were also higher in the TO-group. However, the repeated PCI rate was similar in both groups at clinical follow-up periods of one-month and 12-months.

Multivariate analyses: After adjusting for confounding factors, presenting with TO of the culprit artery in patients with NSTEMI predicted 12-month all-cause mortality (hazard ratio [HR] 2.11, 95\% confidence interval [CI] 1.21 to $3.68, P=$ 0.009 ) (Figure 2). Other predictive factors associated with increased 12-month mortality were prior diabetes mellitus (HR $1.95,95 \%$ CI 1.09 to $3.47, P=0.023$ ), mechanical ventilation (HR $4.42,95 \%$ CI 1.27 to $15.37, P=0.020$ ), left ventricular ejection fraction $\leq 40 \%$ (HR 5.19, 95\% CI 2.95 to $9.13, P<$ $0.001)$, and creatinine clearance $\leq 60 \mathrm{~mL} /$ minute $/ 1.73 \mathrm{~m}^{2}(\mathrm{HR}$ $4.69,95 \%$ CI 2.51 to $8.76, P<0.001$ ).

In subgroup analysis with 930 patients who underwent PCI within one day after admission, in-hospital mortality and 12-month mortality were higher in the TO group (in-hospital mortality; $4.9 \%$ versus $1.4 \%, P=0.005$, and 12 -month mortality; $9.6 \%$ versus $4.1 \%, P=0.004$ respectively).

\section{Discussion}

The results of the present study strongly suggest that TO of the culprit artery in patients with NSTEMI who underwent an early invasive strategy with PCI was associated with increased 12-month mortality. Few studies have compared a TO group and non-TO group in patients with NSTEMI. Wang, et $a l^{4)}$ examined NSTE acute coronary syndrome patients with TO of the culprit artery. About one-fourth had an occlusive culprit artery and culprit lesions were more frequently identified in the inferolateral myocardial territory. Patients with TO of the culprit artery had higher risk-adjusted 6-month mortality. In another study, 448 patients with NSTEMI with TO of the culprit artery were analyzed and 6-month MACE was found to be significantly higher in the TO group than in the non-TO group. ${ }^{5)}$ In the present study, the large population size and characteristics of all enrolled patients who underwent early invasive strategy with PCI provided additional valuable information about the management of patients with NSTEMI with TO of the IRA. In patients with TO among NSTEMI patients who underwent early invasive strategy with PCI, intensive medical therapy with frequent follow-up was needed to reduce mortality during short and long-term follow- up.

As in 2 recent studies, the present study showed LCX and RCA were more common IRA in the TO group. ${ }^{4,5)}$ However, there are no standard methods for predicting IRA in NSTEMI, unlike STEMI, and there are no known factors predicting TO of the IRA in NSTEMI. Multivariate analysis in the present study revealed initial electrocardiographic findings which showed ST-deviation or T-inversion on inferolateral leads and dyslipidemia were factors associated with TO of the IRA in our registry. Although symptom-to-PCI time was not associated with TO, it is known to be associated with TO. ${ }^{3)}$ Because our study population had early PCI, further study is needed to evaluate patients who underwent PCI at various different times.

Our results did not suggest there was any difference in cardiac biomarkers indicating infarct size and left ventricular ejection fraction. However, it is not sufficient to evaluate infarct size by 2-dimensional echocardiograms and cardiac biomarkers. Also, there is no clear evidence that TO of the IRA in patients with NSTEMI had a larger infarct size. Thus, newer technologies such as cardiac magnetic resonance imaging will be needed to confirm this. ${ }^{13)}$

Generally, patients with CAD having LAD as a culprit vessel are known to have worse clinical outcomes (examples; cardiogenic shock) compared with patients with an LCX or RCA culprit artery. In the present study, the TO group had more patients with a non-LAD culprit artery. This finding suggests that other factors were involved in the higher MACE and mortality rates in the TO group. Although post-MI complica- 
tions such as ischemic mitral regurgitation or right ventricular infarction would have a detrimental impact on clinical outcomes in the TO group, our registry unfortunately did not have this information. In-hospital and follow-up 2-dimensional echocardiography would provide additional information. Also, the higher in-hospital complication rate in the TO group was associated with various factors. In our results, the lower PCI success rate and stenting rate, and higher rate of complex lesion in the TO group might be associated with in-hospital events.

Even though STEMI is known to be associated with higher in-hospital mortality, the long-term clinical outcomes are similar regardless of the presence of ST-elevation. ${ }^{14,15)}$ Also, in the KAMIR, 12-month mortality was similar in both STEMI and NSTEMI patients. ${ }^{16)}$ How about the prognosis in NSTEMI patients with TO of the culprit artery? Should NSTEMI patients with TO in the IRA be considered STEMIequivalents? In our opinion, there are difficulties with respect to comparing these two heterogenous groups. To understand this problem, further evaluation of the pathophysiology of TO in NSTEMI patients should be undertaken.

Our study has several limitations. First, it was not a randomized-control trial, and selection bias might exist. Second, no standard method was present in the selection of culprit vessels, especially patients with NSTEMI. Therefore, selection of a culprit vessel under similar conditions might be different among operators. Third, our registry did not have information on collateral circulation in coronary angiograms. Although complex lesions were more commonly seen in the TO group, further angiographic studies are needed. Collateral flows over an occlusive lesion may have affected the clinical outcomes in our patients. Fourth, despite multivariate analysis, predictive factors associated with TO of the IRA were not precisely evaluated. If we can evaluate these factors, the prognosis of these high-risk patients would improve. Finally, although we adjusted for all possible confounding factors, other potent confounding factors were possibly associated with clinical outcomes.

In conclusion, despite these limitations, our study proves patients with NSTEMI with TO of the culprit artery had higher short- and long-term mortality than the non-TO group. To achieve better clinical outcomes in these high-risk patients, predictive factors of TO of the culprit artery should be developed.

\section{APPENDIX}

The KAMIR Investigators: Myung Ho Jeong, Young Jo Kim, Chong Jin Kim, Myeong Chan Cho, Youngkeun Ahn, Jong Hyun Kim, Shung Chull Chae, Seung Ho Hur, In Whan Seong, Taek Jong Hong, Dong Hoon Choi, Jei Keon Chae, Jae Young Rhew, Doo Il Kim, In Ho Chae, Jung Han Yoon, Bon Kwon Koo, Byung Ok Kim, Myoung Yong Lee, Kee Sik Kim, Jin Yong Hwang, Seok Kyu Oh, Nae Hee Lee, Kyoung Tae Jeong Seung Jea Thak, Jang Ho Bae, Seung Woon Rha, Keum Soo Park, Kyoo Rok Han, Tae Hoon Ahn, Moo Hyun Kim, Joo Young Yang, Chong Yun Rhim, Hyeon Cheol Gwon, Soo Joong Kim, Dong Kyu Jin, Jin Man Cho, Wook Sung Chung, Yang Soo Jang, Jeong Gwan Cho, Ki Bae Seung, and Seung Jung Park.

\section{REFERENCES}

1. Anderson JL, Adams CD, Antman EM, et al. ACC/AHA 2007 guidelines for the management of patients with unstable angina/ non-ST-elevation myocardial infarction: a report of the American College of Cardiology/American Heart Association Task Force on Practice Guidelines. J Am Coll Cardiol 2007; 50: e1-e157.

2. Antman EM, Anbe DT, Armstrong PW, et al. ACC/AHA guidelines for the management of patients with ST-elevation myocardial infarction. Circulation 2004; 110: 588-636.

3. DeWood MA, Stifter WF, Simpson CS, et al. Coronary arteriographic findings soon after non-Q-wave myocardial infarction. N Engl J Med 1986; 315: 417-23.

4. Wang TY, Zhang M, Fu Y, et al. Incidence, distribution, and prognostic impact of occluded culprit arteries among patients with non-ST-elevation acute coronary syndromes undergoing diagnostic angiography. Am Heart J 2009; 157: 716-23.

5. Bahrmann P, Rach J, Desch S, Schuler GC, Thiele H. Incidence and distribution of occluded culprit arteries and impact of coronary collaterals on outcome in patients with non-ST-segment elevation myocardial infarction and early invasive treatment strategy. Clin Res Cardiol 2010; 100: 457-67.

6. Manginas A, Gatzov P, Chasikidis C, Voudris V, Pavlides G, Cokkinos DV. Estimation of coronary flow reserve using the Thrombolysis in Myocardial Infarction (TIMI) frame count method. Am J Cardiol 1999; 83: 1562-5. (Review)

7. Myocardial infarction redefined--a consensus document of The Joint European Society of Cardiology/American College of Cardiology Committee for the redefinition of myocardial infarction. Eur Heart J 2000; 21: 1502-13. (Review)

8. Antman EM, Cohen M, Bernink PJ, et al. The TIMI risk score for unstable angina/non-ST elevation MI: A method for prognostication and therapeutic decision making. JAMA 2000; 284: 835-42.

9. Gibson CM, Schömig A. Coronary and myocardial angiography: angiographic assessment of both epicardial and myocardial perfusion. Circulation 2004; 109: 3096-105. (Review)

10. Thygesen K, Alpert JS, White HD; Joint ESC/ACCF/AHA/WHF Task Force for the Redefinition of Myocardial Infarction. Universal definition of myocardial infarction. Eur Heart J 2007; 28 : 2525-38

11. Cutlip DE, Windecker S, Mehran R, et al. Clinical end points in coronary stent trials: a case for standardized definitions. Circulation 2007; 115: 2344-51.

12. Lee JH, Yang DH, Park HS, et al. Suboptimal use of evidencebased medical therapy in patients with acute myocardial infarction from the Korea Acute Myocardial Infarction Registry: prescription rate, predictors, and prognostic value. Am Heart J 2010; 159: 1012-9.

13. Klocke FJ. Cardiac magnetic resonance measurements of area at risk and infarct size in ischemic syndromes. J Am Coll Cardiol 2010; 55: 2489-90.

14. Montalescot G, Dallongeville J, Van Belle E, et al. STEMI and NSTEMI: are they so different? 1 year outcomes in acute myocardial infarction as defined by the ESC/ACC definition (the OPERA registry). Eur Heart J 2007; 28: 1409-17.

15. Abbott JD, Ahmed HN, Vlachos HA, Selzer F, Williams DO. Comparison of outcome in patients with ST-elevation versus non-ST-elevation acute myocardial infarction treated with percutaneous coronary intervention (from the National Heart, Lung, and Blood Institute Dynamic Registry). Am J Cardiol 2007; 100: 190-5.

16. Sim DS, Jeong MH, Kang JC. Current management of acute myocardial infarction: experience from the Korea Acute Myocardial Infarction Registry. J Cardiol 2010; 56: 1-7. (Review) 